\title{
Homozygous deletion of the HLA-B gene as an acquired- resistance mechanism to nivolumab in a patient with lung adenocarcinoma: a case report
}

\author{
He Zhang ${ }^{1 \#}$, Weiwei Dong ${ }^{1 \#}$, Huixia Zhao ${ }^{1}$, Zhiyan Zeng ${ }^{1}$, Fengyun Zhang ${ }^{1}$, Yanyan $\mathrm{Hu}^{1}$, Qiuwen $\mathrm{Li}^{1}$, \\ Jing Chen ${ }^{2}$, Erhong Meng ${ }^{2} \wedge$, Wenhua Xiao ${ }^{1}$ \\ ${ }^{1}$ Department of Oncology, The Fifth Medical Center, Chinese PLA General Hospital, Beijing, China; ${ }^{2}$ ChosenMed Technology (Beijing) Co. Ltd, \\ Beijing, China \\ \#These authors contributed equally to this work and share first authorship. \\ Correspondence to: Erhong Meng. ChosenMed Technology (Beijing) Co. Ltd, Beijing, China. Email: erhongmeng@chosenmedtech.com; Wenhua \\ Xiao. Department of Oncology, The Fifth Medical Center, Chinese PLA General Hospital, Beijing, China. Email: w_hxiao@hotmail.com.
}

\begin{abstract}
Immune checkpoint inhibitors (ICIs) have greatly improved the treatment of advanced nonsmall-cell lung cancer, including lung adenocarcinoma (LUAD). Patients treated with ICIs can have longterm clinical outcomes; however, acquired resistance to ICI therapy has been frequently observed. To date, little is known about the underlying mechanisms. In this study, we report the case of a male smoker with metastatic LUAD who initially received multi-line radiotherapy and chemotherapy and achieved stable disease (SD) for almost 10 years. The patient was treated with nivolumab for about 15 months. However, the disease later progressed rapidly. A genetic profile of the patient revealed the homozygous deletion of the human leukocyte antigen $(H L A)-B$ gene, which may have conferred the acquired resistance. Our study is the first to describe the homozygous deletion of the $H L A-B$ gene as an acquired-resistance mechanism to programmed cell death protein 1 (PD-1) blockade in a patient with LUAD. This evidence suggests that tumor cells can selectively lose $H L A-A, B$, and $C$ to survive under strong immune pressure. This discovery enriches and develops our understanding of the mechanism of drug resistance in ICI therapy in LUAD. However, further investigations are urgently needed to be conducted to determine how this resistance can be overcome.
\end{abstract}

Keywords: Lung adenocarcinoma (LUAD); immune checkpoint inhibitors (ICIs); acquired resistance; homozygous deletion of HLA-B; case report

Submitted Jul 02, 2021. Accepted for publication Aug 30, 2021.

doi: 10.21037/atm-21-3825

View this article at: https://dx.doi.org/10.21037/atm-21-3825

\section{Introduction}

Lung adenocarcinoma (LUAD) is the most common subtype of non-small cell lung cancer (NSCLC) (1) and accounts for the majority of cancer-related deaths worldwide (2). In recent years, immunotherapy based on immune checkpoint inhibitors (ICIs) has greatly improved the survival of patients with driver gene-negative LUAD.
However, the biggest barrier to effective immunotherapy is primary or acquired resistance (3). To maximize the clinical benefits of ICI therapy, it is essential to clarify the mechanism underlying the resistance.

Currently, studies have reported that the putative mechanism of resistance includes variations of functional genes and changes in the tumor microenvironment (4).

^ ORCID: 0000-0002-4573-3430. 
Research has shown that the anti-tumor activity of ICIs rely on CD8+ T cell, MHC-I-dependent immune activity (5-8). The MHC-I complex comprises a heavy chain encoded by the $H L A-A,-B$, and $-C$ genes and a light chain $\beta 2-$ microglobulin ( $\beta 2 M)(9)$. Mutations in any of the abovementioned genes can affect patients' response to ICI treatment (10).

Recently, defective HLA-I molecules have been reported to be associated with immune escape and to be negative prognostic factors of immunotherapy in multiple cancer types (8,9,11-16). Rodig et al. found the majority or complete deletion of the MHC-I membrane was associated with primary resistance to CTLA-4 in melanoma (17). In addition, Paulson et al. reported that the transcriptional loss of HLA-I may underlie the acquired resistance to combined immunotherapies, including ICIs, in metastatic Merkel cell carcinoma (18). The association between HLA-I deficiency and immune escape in lung cancer has also been investigated. McGranahan et al. observed that $40 \%$ of early stage NSCLC showed a HLA loss of heterozygosity $(\mathrm{LOH})$, which is an immune escape mechanism (12). Further, using human lung cancer cell lines, Perea $e t$ al. reported that a $\mathrm{LOH}$ (with a complete or partial loss of the HLA-I genes) was the main mechanism underlying HLA-I variations, and could potentially reduce the clinical efficacy of immunotherapy in lung cancer (11). In addition, $\beta 2 \mathrm{M}$ is the light chain and a component part of the HLA class-I molecule. Gettinger et al. found that the knockout of $\beta 2 M$ gene via clustered regularly interspaced short palindromic repeats (CRISPR) in a lung cancer mouse model disrupted HLA-I antigen processing as well as the presentation machinery, and mediated immune escape from ICIs (19).

However, to date, no research appears to have been conducted on whether the homozygous deletion of the $H L A-B$ gene contributes to acquire ICI resistance in LUAD.

In this study, we report the case of a 54-year-old male patient with LUAD, who is a heavy cigarette smoker. He initially received multi-line radiotherapy and chemotherapy, followed by treatment with nivolumab. He achieved stable disease (SD) for about 15 months after treatment with the programmed cell death protein 1 (PD-1) inhibitor. However, the disease later progressed. Next-generation sequencing (NGS) revealed the homozygous deletion of the human leukocyte antigen $(H L A)-B$ gene. To the best of our knowledge, this is the first study to report on the homozygous deletion of the $H L A-B$ gene in a patient with LUAD who developed acquired resistance to the PD-1 blockade. We present the following case in accordance with the CARE reporting checklist (available at https://dx.doi. org/10.21037/atm-21-3825).

\section{Case presentation}

The study conformed to the provisions of the Declaration of Helsinki (as revised in 2013). This study was approved by the Ethics Committee of The Fifth Medical Center of the Chinese PLA General Hospital. Written informed consent was obtained from the patient for publication of this case report and accompanying images. A copy of the written consent is available for review by the editorial office of this journal.

On November 18, 2011, during a physical examination, a thoracic computed tomography (CT) scan showed spaceoccupying lesions on the left upper pulmonary lobe of a 54-year-old male patient, indicating that the peripheral lung cancer had metastasized to the mediastinal lymph node. On November 21, 2011, a thoracic CT at the Cancer Hospital of Chinese Academy of Medical Sciences revealed that the lesion was approximately $2.2 \mathrm{~cm} \times 3.2 \mathrm{~cm} \times 5 \mathrm{~cm}$ in size. Spreading to the lymph node was found in the bilateral mediastinum area 4-6 and the left hilar, and the shortest diameter was about $2.5 \mathrm{~cm}$. On November 29 , 2011, brush cytology and a lymph node biopsy at station 4R were performed, and adenocarcinoma cells were detected. Amplification-refractory mutation system and Ventana assay showed that both the epidermal growth factor receptor $(E G F R)$ and anaplastic lymphoma kinase $(A L K)$ mutation, respectively, were negative. The tumor adhered closely to the aortic arch, which indicated that it may have invaded the aorta. The final diagnosis was stage IIIB poorly differentiated LUAD (pT4N3M0).

The patient was then admitted to our department for local radiotherapy of the bilateral lung, mediastinum and bilateral supraclavicular lymphatic drainage area. $\mathrm{He}$ was also treated with $90 \mathrm{mg}$ of paclitaxel per week for sensitization. On January 14, 2012, he underwent docetaxel plus cisplatin-based chemotherapy for 3 cycles. As suspicious spreading to the liver was observed, the chemotherapy regimen was changed to gemcitabine, cisplatin, and endostar for 2 cycles. In March 2015 and June 2016, chest CT scans showed the lung lesion was larger. On August 15, 2016, a positron emission tomography/CT scan showed left upper lobe nodules, measuring about $2.8 \mathrm{~cm} \times 3.3 \mathrm{~cm}$ in size. The diameter of the supraclavicular lymph node was approximately $6 \mathrm{~mm} \times 9 \mathrm{~mm}$, indicating that the lung cancer was still active; however, there was no evidence of metastasis 

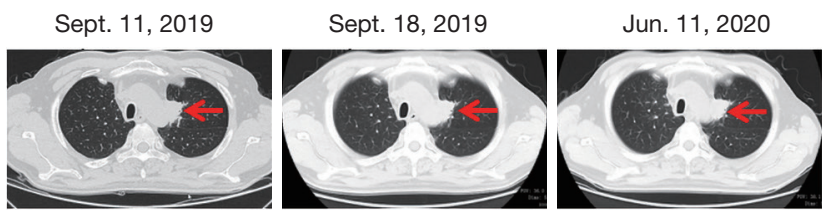

Dec. 09, 2020
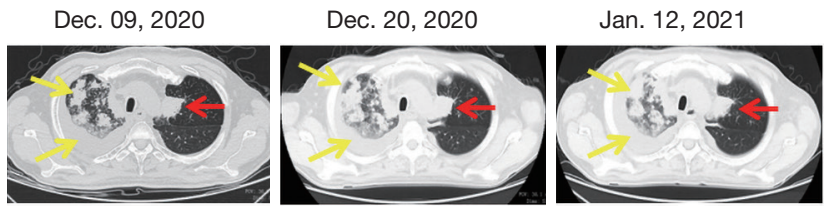

Figure 1 Dynamic CT imaging of pulmonary lesions at multiple time points. The red arrow indicates the lesion in the left lung, while the yellow arrows indicate the metastatic lesions and pleural effusions in the right lung. No metastasis in the right lung was detected before resistance to nivolumab; however, after resistance to nivolumab, the metastasis in the right lung increased greatly, and bilateral effusion, especially right pleural effusion, developed.

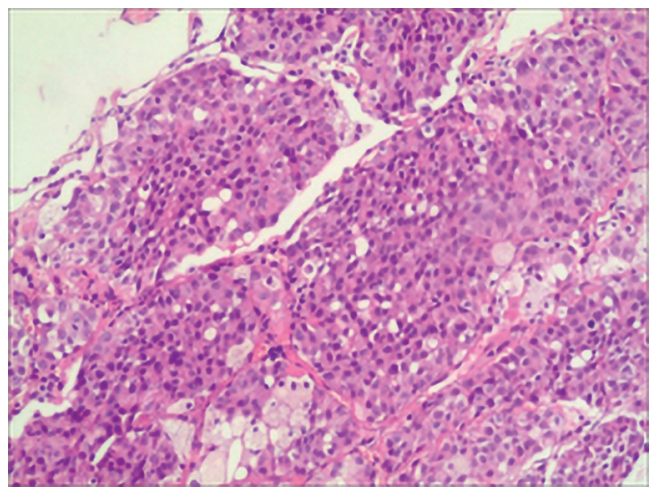

Figure 2 Pathology of tumor tissues from the right lung revealed poorly differentiated adenocarcinoma. Hematoxylin-eosin staining, magnification: $\times 200$.

to other regions. The peripheral blood circulating tumor cell was $11.15 \mathrm{FU} / 3 \mathrm{~mL}$ (reference range: $0-8.7 \mathrm{FU} / 3 \mathrm{~mL}$ ). From May 2017, the patient received 2 cycles of pemetrexed disodium plus cisplatin. On March 22, 2019, a thoracic CT scan showed that the tumor was larger, indicating progressive disease (PD). From March 19, 2019 to December 15, 2019, the patient received combination therapy of pemetrexed disodium plus nivolumab, and the disease was evaluated as SD (see Figure 1).

Due to COVID-19, the patient was treated with nivolumab monotherapy for maintenance until June 2020 at a local hospital. In June 2020, due to the interstitial changes in the right lung and a suspicion that these changes were caused by immune-related adverse events, the patient was taken off nivolumab and began hormone therapy. As the lesion in the right lung progressed, on December 9, 2020 an ultrasound-guided needle biopsy of the lesion in the right lung was performed to clarify the nature of the lesion. The pathology results confirmed a diagnosis of poorly differentiated adenocarcinoma (see Figure 2). Immunohistochemical (IHC) staining revealed that the tumor tissues were positive for cytokeratin $8 / 18$, thyroid transcription factor-1, Ki-67 (30\%), MLH1, MSH2, MSH 6 and PMS2, but negative for P40, CD56 or synaptophysin. The disease was evaluated as PD.

On December 21, 2020 and January 12, 2021, the patient received chemotherapy with albumin-bound paclitaxel and lobaplatin twice. Due to tightness in the chest and shortness of breath when lying down, pleural effusion drainage and intrapleural perfusion with lobaplatin plus endostar were performed. A chest CT scan revealed multiple nodules and mass shadows in both lungs, which suggested metastasis. The lesion in the right upper lobe of the lung was more progressive than before. Further, moderate pericardial effusion and bilateral effusion, especially right pleural effusion, were detected, indicating disease progression (see Figure 1). From February 3, 2020, the patient was placed on irinotecan and achieved SD, but without any obvious improvement. Figure 3 details the course of treatment.

To evaluate potential therapeutic strategies, on December 11, 2020, NGS through a pan-cancer 599-gene panel [ChosenOne599, ChosenMed Technology (Beijing) Co. Ltd, Beijing, China] was performed, and the results revealed a high tumor mutational burden (TMB-H, 21.48 Muts/Mb) and high microsatellite instability (MSI-H). Notably, the homozygous deletion of the $H L A-B$ gene was observed (see Table 1 and Figure 4).

\section{Discussion}

Currently, our understanding of the mechanism underlying the primary and acquired resistance to ICI therapy in LUAD is limited. In this study, we identified the homozygous deletion of the $H L A-B$ gene in a patient with LUAD who developed an acquired resistance to nivolumab. This study is the first one to report on the homozygous deletion of the $H L A-B$ gene as an acquired-resistance mechanism to the PD-1 inhibitor in LUAD. 


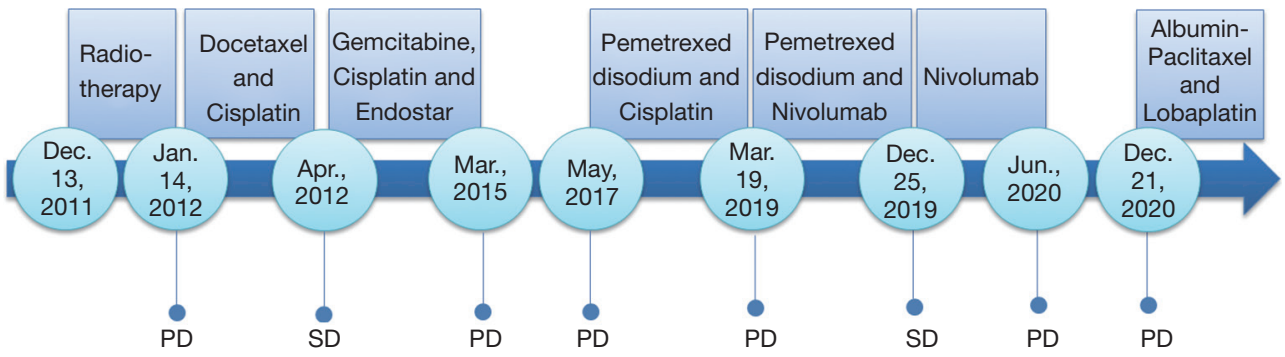

Figure 3 Course of treatment. PD, progressive disease; SD, stable disease.

Table 1 Genomic profile of the patient

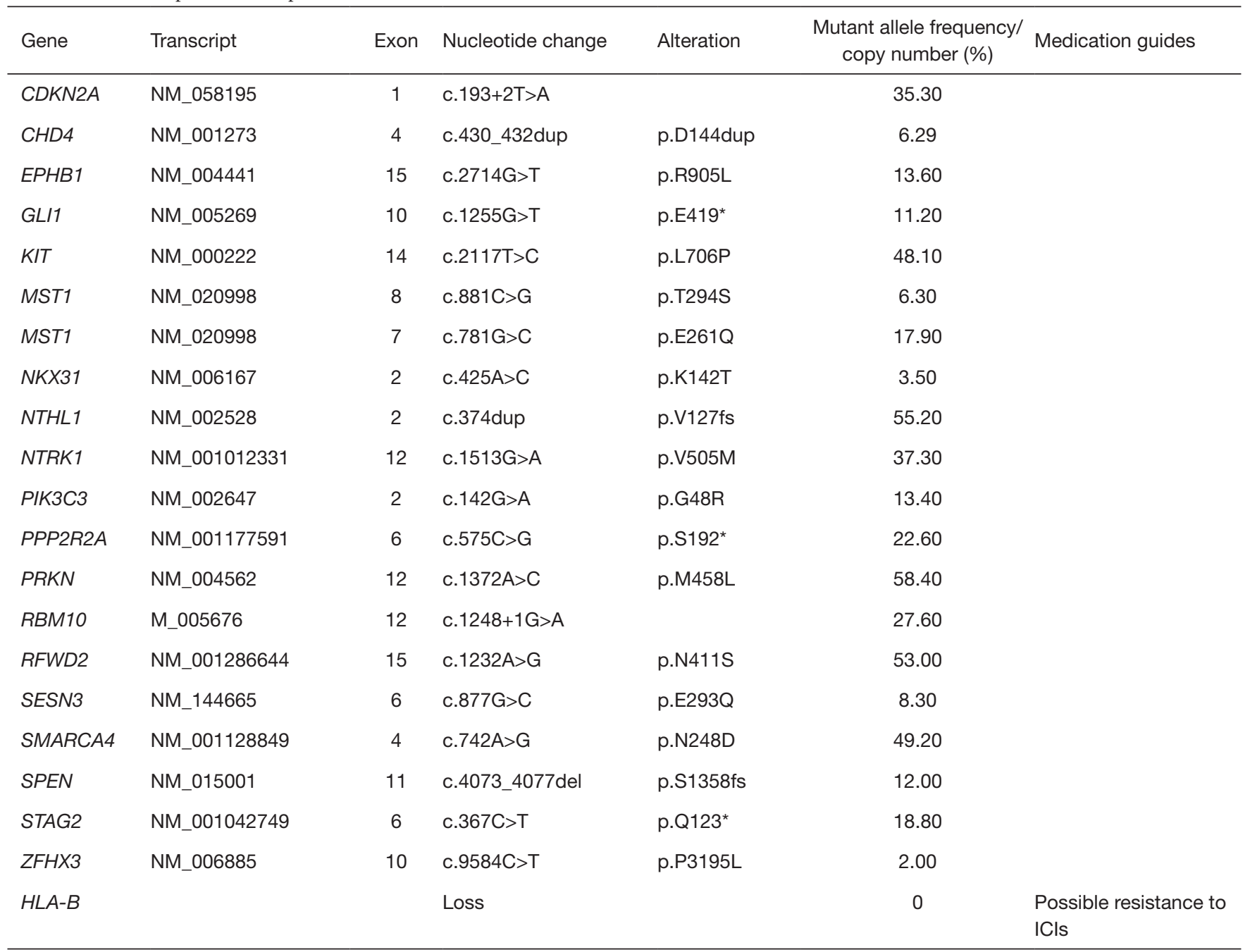

The patient in the present study achieved SD after receiving nivolumab for approximately 15 months. However, metastasis to the right lung was subsequently discovered. The NGS revealed a TMB-H and MSI-H.
Additionally, IHC staining revealed the positive expression of PD-L1. Together, these results indicated that the patient should be sensitive to ICIs. However, the finding that the gene copy number of the $H L A-B$ was 0 in the patient 

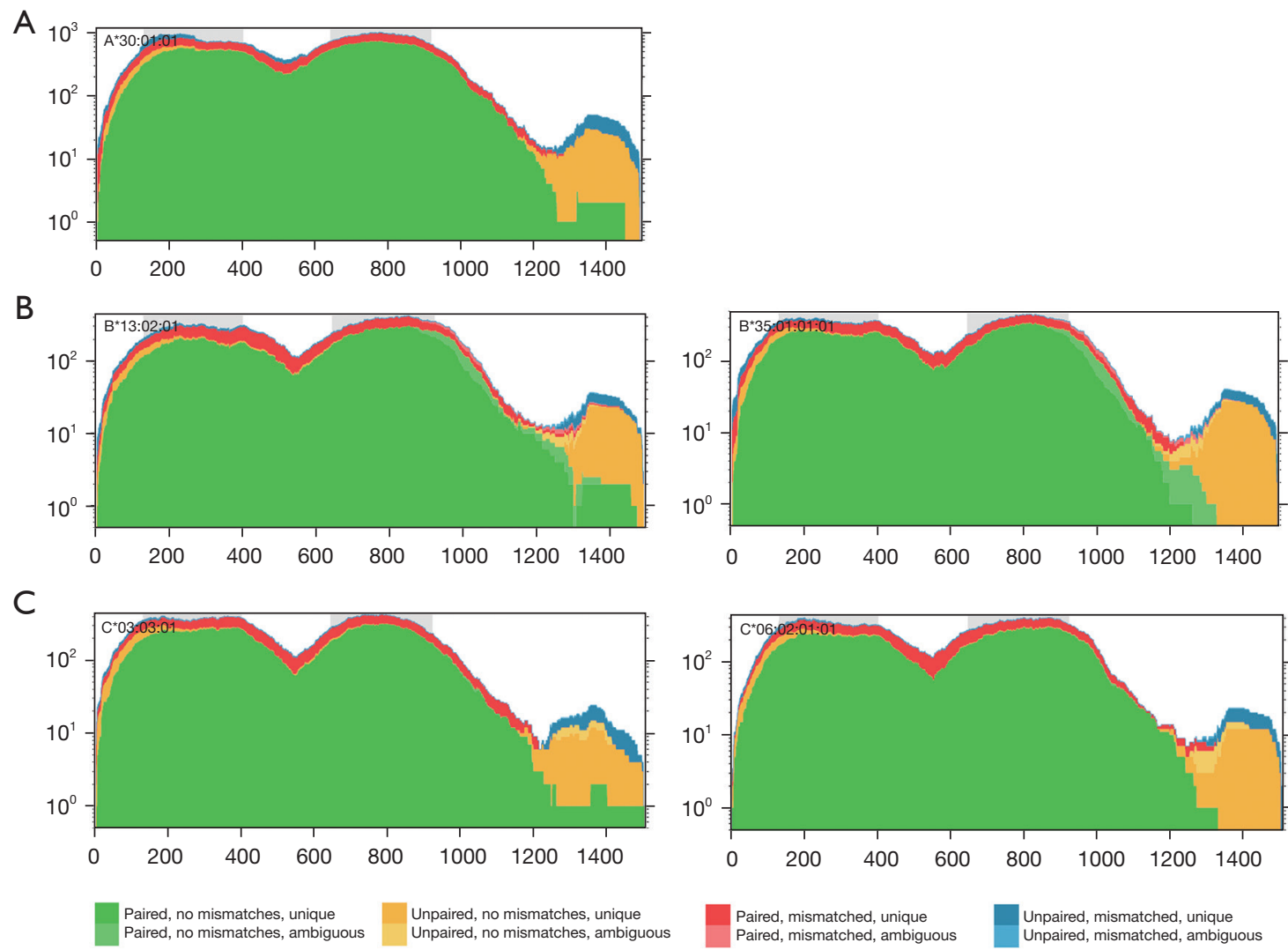

Figure 4 Loss of the $H L A-B$ gene illustrated by NGS in the patient. There was no deletion of the $H L A-A$ (A) or $H L A-C$ gene (C), but the loss of the $H L A-B$ gene was detected in the patient by NGS (B). NGS, next-generation sequencing.

negated all the above favorable indicators. $H L A-B$ is an essential component of the MHC-I complex. In the present case, the homozygous deletion of the $H L A-B$ gene led to defective HLA-I molecules, abnormal antigen processing and aberrant antigen presentation to CD8+ cells. The other mutations observed in this patient, such as the $C D K N 2 A$, $C H D 4$, and EPHB1 mutations, have not yet been reported to be related to ICI resistance; thus, the homozygous deletion of the $H L A-B$ gene conferred a resistance to nivolumab in this patient. This is consistent with the discovery of Perea et al. that HLA-I loss was related to a greatly decreased density of immune infiltration (11).

Most human cells contain two sets of HLA genes; one of which is inherited from the mother, and the other, from the father. HLA LOH refers to the complete or partial deletion of the paternal or maternal HLA haplotype (12). Previous research has reported that HLA LOH is the most common mechanism of HLA haplotype absence in malignancies. Yan et al. discovered that the resistance to ICIs by HLA LOH could be reversed by a low-dose decitabine with PD-1 inhibitors. It may be that histone deacetylase inhibitors increase the expression of MHC and tumor antigen, and enhance the infiltration of CD8+, CD4+, and IFN $\gamma+\mathrm{T}$ cells (13-16,20). However, in our study, we observed the homozygous deletion of the $H L A-B$ gene; thus, low-dose decitabine combination with PD-1 inhibitors might not work.

Our findings indicate that tumor cells can selectively lose $H L A-A, B$ and $C$ genes to survive under strong immune pressure. Further, our results provide additional evidence that abnormal antigen processing and presentation contribute to the acquired resistance to ICIs in LUAD. This discovery enriches and develops current understandings of the mechanism of drug resistance in immunotherapy in LUAD.

Additionally, IHC staining showed proficient mismatch repair in the patient, while NGS revealed that the patient harbored MSI-H, which is very rare in lung cancer. Thus, NGS effectively detected the unstable state of microsatellites, which greatly made up for the shortcomings of traditional detection methods.

A limitation of this study is that no effective therapeutic 
strategy was found to address the acquired resistance.

\section{Conclusions}

This study is the first to report on the homozygous deletion of the $H L A-B$ gene arising from an acquired-resistance mechanism to the PD-1 blockade in a patient with LUAD. Further investigations urgently need to be conducted to determine how this resistance can be overcome.

\section{Acknowledgments}

The authors would like to thank the patient and all of the personnel for their participation in this study.

Funding: None.

\section{Footnote}

Reporting Checklist: The authors have completed the CARE reporting checklist. Available at https://dx.doi. org/10.21037/atm-21-3825

Conflicts of Interest: All authors have completed the ICMJE uniform disclosure form (available at https://dx.doi. org/10.21037/atm-21-3825). JC and EM are employees of ChosenMed Technology. The other authors have no conflicts of interest to declare.

Ethics Statement: The authors are accountable for all aspects of the work in ensuring that questions related to the accuracy or integrity of any part of the work are appropriately investigated and resolved. The study conformed to the provisions of the Declaration of Helsinki (as revised in 2013). This study was approved by the Ethics Committee of The Fifth Medical Center of the Chinese PLA General Hospital. Written informed consent was obtained from the patient for publication of this case report and accompanying images. A copy of the written consent is available for review by the editorial office of this journal.

Open Access Statement: This is an Open Access article distributed in accordance with the Creative Commons Attribution-NonCommercial-NoDerivs 4.0 International License (CC BY-NC-ND 4.0), which permits the noncommercial replication and distribution of the article with the strict proviso that no changes or edits are made and the original work is properly cited (including links to both the formal publication through the relevant DOI and the license).
See: https://creativecommons.org/licenses/by-nc-nd/4.0/.

\section{References}

1. Alam H, Li N, Dhar SS, et al. HP1 $\gamma$ Promotes Lung Adenocarcinoma by Downregulating the TranscriptionRepressive Regulators NCOR2 and ZBTB7A. Cancer Res 2018;78:3834-48.

2. Bray F, Ferlay J, Soerjomataram I, et al. Global cancer statistics 2018: GLOBOCAN estimates of incidence and mortality worldwide for 36 cancers in 185 countries. CA Cancer J Clin 2018;68:394-424.

3. Kocher F, Amann A, Zimmer K, et al. High indoleamine2,3-dioxygenase 1 (IDO) activity is linked to primary resistance to immunotherapy in non-small cell lung cancer (NSCLC). Transl Lung Cancer Res 2021;10:304-13.

4. Sharma P, Hu-Lieskovan S, Wargo JA, et al. Primary, Adaptive, and Acquired Resistance to Cancer Immunotherapy. Cell 2017;168:707-23.

5. Gubin MM, Zhang X, Schuster H, et al. Checkpoint blockade cancer immunotherapy targets tumour-specific mutant antigens. Nature 2014;515:577-81.

6. Jiang H, Zhang T, Yan MX, et al. IL-35 inhibits CD8+ T cells activity by suppressing expression of costimulatory molecule CD28 and Th1 cytokine production. Transl Cancer Res 2019;8:1319-25.

7. Tran E, Robbins PF, Lu YC, et al. T-Cell Transfer Therapy Targeting Mutant KRAS in Cancer. N Engl J Med 2016;375:2255-62.

8. Chowell D, Morris LGT, Grigg CM, et al. Patient HLA class I genotype influences cancer response to checkpoint blockade immunotherapy. Science 2018;359:582-7.

9. Cornel AM, Mimpen IL, Nierkens S. MHC Class I Downregulation in Cancer: Underlying Mechanisms and Potential Targets for Cancer Immunotherapy. Cancers (Basel) 2020;12:1760.

10. Conway JR, Kofman E, Mo SS, et al. Genomics of response to immune checkpoint therapies for cancer: implications for precision medicine. Genome Med 2018;10:93.

11. Perea F, Sánchez-Palencia A, Gómez-Morales M, et al. HLA class I loss and PD-L1 expression in lung cancer: impact on T-cell infiltration and immune escape. Oncotarget 2018;9:4120-33.

12. McGranahan N, Rosenthal R, Hiley CT, et al. AlleleSpecific HLA Loss and Immune Escape in Lung Cancer Evolution. Cell 2017;171:1259-1271.e11.

13. Yan X, Zhao Y, Liu Y, et al. Case Report: Low-Dose 
Decitabine Plus Anti-PD-1 Inhibitor Camrelizumab for Previously Treated Advanced Metastatic Non-Small Cell Lung Cancer. Front Oncol 2020;10:558572.

14. Nie J, Zhang Y, Li X, et al. DNA demethylating agent decitabine broadens the peripheral $T$ cell receptor repertoire. Oncotarget 2016;7:37882-92.

15. Vo DD, Prins RM, Begley JL, et al. Enhanced antitumor activity induced by adoptive $\mathrm{T}$-cell transfer and adjunctive use of the histone deacetylase inhibitor LAQ824. Cancer Res 2009;69:8693-9.

16. Chen M, Nie J, Liu Y, et al. Phase Ib/II study of safety and efficacy of low-dose decitabine-primed chemoimmunotherapy in patients with drug-resistant relapsed/refractory alimentary tract cancer. Int J Cancer 2018;143:1530-40.

17. Rodig SJ, Gusenleitner D, Jackson DG, et al. MHC proteins confer differential sensitivity to CTLA-4 and

Cite this article as: Zhang $\mathrm{H}$, Dong $W$, Zhao $\mathrm{H}$, Zeng Z, Zhang F, Hu Y, Li Q, Chen J, Meng E, Xiao W. Homozygous deletion of the $H L A-B$ gene as an acquiredresistance mechanism to nivolumab in a patient with lung adenocarcinoma: a case report. Ann Transl Med 2021;9(19):1506. doi: 10.21037/atm-21-3825
PD-1 blockade in untreated metastatic melanoma. Sci Transl Med 2018;10:eaar3342.

18. Paulson KG, Voillet V, McAfee MS, et al. Acquired cancer resistance to combination immunotherapy from transcriptional loss of class I HLA. Nat Commun 2018;9:3868.

19. Gettinger S, Choi J, Hastings K, et al. Impaired HLA Class I Antigen Processing and Presentation as a Mechanism of Acquired Resistance to Immune Checkpoint Inhibitors in Lung Cancer. Cancer Discov 2017;7:1420-35.

20. Li X, Zhang Y, Chen M, et al. Increased IFN $\gamma+$ T Cells Are Responsible for the Clinical Responses of LowDose DNA-Demethylating Agent Decitabine Antitumor Therapy. Clin Cancer Res 2017;23:6031-43.

(English Language Editor: L. Huleatt) 